\title{
Review on the Modern portfolio theory and optimization model
}

\author{
Zehan $\mathrm{Hou}^{1, \mathrm{a}}$, Zeyu $\mathrm{Li}^{2, \mathrm{~b}}$ and Yang Zhou ${ }^{3, \mathrm{c}}$ \\ ${ }^{1}$ Durham University Business School, Durham University, Durham City DH1 3DE,China \\ ${ }^{2}$ School of Economics, Sichuan University, Chengdu 610065, China \\ ${ }^{3}$ Bayi international department, Beijing Bayi School, Beijing 100080, China \\ a'Garfieldhou@163.com, beyu_li@outlook.com, cm18311139290@163.com
}

Keywords: Modern Portfolio Theory (MPT), Indexed, Improved, Essential and Dynamic.

\begin{abstract}
The field of portfolio optimization and risk management are the bases to develop and improve the financial markets and also the financial decisions. It has narrow and broad part of theory. A narrow theory refers to the Modern portfolio theory (MPT); and another version of the theory generalizes the classical theory to accommodate various alternative theories, including the capital asset pricing model and effective securities market theory of capital market theory. At the same time, because of the traditional efficient market hypothesis cannot explain market anomalies, the portfolio theory is facing the challenge of irrational market movements. Then behavioral finance was introduced to tackle the problem. The Topic of modern portfolio theory and the optimization model of development are reviewed in this paper. The analytical models outline the development trend of related fields, pointing out the direction for future research. The ideas introduced in this article forms the foundations of what is now popularly referred to as Modern Portfolio Theory (MPT).
\end{abstract}

\section{Introduction}

The financial community strongly adopted the MPT, and now 50 years later, MPT generated relatively great interest. Based on those very same principles new models are constantly being reinvented to incorporate all the new findings that result from that seminal work. In the present research, the improvement of portfolio mean variance Model has been focused on the Sharp Ratio. The examination has displayed a steady model of portfolio in which the consequences of the advancement portfolio have been appeared by the important comparing stable formulizing system. It involves the Factor Model by utilizing money markets files as the information and the trial of the security of the info parameters. To do this the information is selected in a given certainty level taking into account the most exceedingly risky situation with a specific end goal to amplify the Sharp Ratio [5].

Issues of choosing portfolio consist of allocation of cash-flows and resources to various assets for a goal of bringing the higher yield with the less risk. The first scientific model of portfolio decision was introduced by the models in [1] [2]. These different models which have impact on financial markets are the fundamental theory for allocation of capital assets and most of them are based on the original model (CAPM) and the theoretical framework proposed by Sharp.

In addition, Modern portfolio theory has shaped the industry of asset management. It is worth noting that portfolio selection is a normative theory, which describes a norm of behavior that investors should pursue in constructing a portfolio, in contrast to the empirical fact that is actually followed [4]. Asset pricing theory such as the capital asset pricing model formalizes the relationship that should exist between asset returns and risk if investors selected portfolios according to mean-variance analysis. In contrast to a normative theory, asset pricing theory is a positive theory-a theory that hypothesizes how investors behave rather than how investors should do [3]. Based on that hypothesized behavior of investors, a model that provides the expected return (a key input of 
constructing portfolios based on mean-variance analysis) is derived and is called an asset pricing model.

Together MPT and asset pricing theory offers a structure to measure investment risk and to create connections between expected asset return and risk. It is critical that Modern portfolio theory is a theory that is different from any theories of asset pricing. Therefore, it should be understood that the validity of modern portfolio theory does not depend on the asset pricing theory, which is not clear to many critics of MPT [7] [8]. Markowitz developed the mean-variance theory as a prescriptive theory, not a descriptive one. Behavioral portfolio theory is descriptive. We note that some investors use procedures that aid in the consideration of co-variances. For example, money managers often apply mean variance optimization and consider Sharpe ratios in the allocation of securities within their funds. But consideration of co-variances within each fund is quite different from consideration of co-variances in the portfolio as a whole. The former leads to suboptimal solutions

\section{Related Work}

The researchers' conclusion to be console base of portfolio determination, today this item is to point by plural of the word same as different normal investigation, different normal improved, and portfolio modern model. At that point of remarkable consideration focuses in Markowitz model, are, regard for risk in contributing not just depend on risk bonds one by one, assume they depend on risk of speculation accumulation. Markowitz model depended on under information: All of entrepreneur has desire expansion alluring, and their attractive breaking point bend is reasoning needy and all of business people are attempting to make most extreme alluring [9]. Financial advisors, such as mutual fund companies, recommend portfolios by a structure of mean variance portfolios in the capital asset pricing model (CAPM). For example, Canner Mankiw and Weil (1997) note that some portfolios be constructed with higher ratios of stocks to bonds than other portfolios, advice that is in conflict with "two-fund separation." Advice consistent with two-fund separation calls for a fixed ratio of stocks to bonds in the "risky" portfolio along with varying properties of the risk-free asset. This reflects varying preference over risk. Canner, Mankiw, and Weil call it "the asset allocation puzzle." Mean-variance investors evaluate portfolios as a whole; they consider co-variances between assets as they construct their portfolios. Mean-variance investors care only about the expected returns and variance of the overall portfolio, not its individual assets. Mean variance investors have consistent attitudes towards risk, they are always averse to risk. Behavioral investors are different. Contrary to the mean-variance, typical investors don't emphasize co-variances and display inconsistent attitudes toward risk. Sharpe notes that while most portfolio optimization programs assume constant attitudes towards risk, experience shows that attitudes are not constant [12].

To put the theory into practical computation, some other numerical assumptions are applied. For example, researchers usually assume the asset returns are Gaussian random variables which follow a normal distribution. However, many studies show that the returns are not normal. In the occasion with a number of benefits, portfolio's effectiveness will use Gaussian distribution [13]. The above description is a circumstance which is not totally common. In this real world for various reasons there are great differences between assets.

In first suspicion, with figuring different as risk standard, by all species helpful and negative possible instability in future, are risk. Unique presentation in this rule is simultaneous themselves respect to incredible productivity of desire effectiveness and little proficiency. Truth be told of vision of financial specialist to take incredible proficiency blessed desire effectiveness not just are not undesired, assume speculator being going to meet, particularly in positively trending market, speculator are taking after looking into acknowledge of top and extraordinary productivity [15]. 
Issue of portfolio decision to create as an example of advanced portfolio. Stable portfolio improved takes this certainty, productivity dispersion to pricing under study in every minute by authentic effectiveness. Unconfident structure idea or non-last nature structure for different and desire effectiveness assessment and they indicated certainties of proficiency of stable portfolio distribution in a specific certainty level are unclear. Making most extreme is more skeptical than sharp regard, one of stable portfolio advanced [10].

The researchers augmented this strategy and they upheld of this arrangement 'picking conservativeness portfolio, has impact when the subsequent to increment in portfolio effectiveness in more negative of situation. These two essayist were been utilizing when in doubt of uncertainty sets of imported parameter for effectiveness dispersion in making model furthermore, they were been utilizing of particular uncertainty sets for appropriation normal and different evaluated. Utilization of stable assessing, $M$ pricing, $S$ assessing with the exception of negative situation and indicated outside specimen particulars in point by point. For more colleagues in these premise late quests, creator can look into exercises research in picking stable portfolio [6].

Specialist added portfolio protection insurance to advanced portfolio with differential coefficient sheets in portfolio stable upgraded senator general structure as covering improper mischance option market. A general entry was created to making upgraded writing, worked exceptional to stable slanted making enhanced of talk in a rundown look [11].

Researchers investigated evaluation of income with utilizing of appraisal respect and pointers for 10 scheme organizations. They did generation to the model for making enhanced portfolio with utilizing of Genetic Programming. In this model, esteem centered in as evaluation risk measure in question risk. The agents did application the created model for surrounding portfolio sorted out of 4 shares of various organizations in stock trade. Results were in premise of risk, when business sector risk making model of proficiency strategy to essential worth proposal are in seethed and Genetic Algorithm advanced technique in taking enhanced weights of shares wicker container with in looking to confinement [14].

Creator did their opportunity to utilizing of partition inevitable space technique to presentation a model for picking the improved portfolio with looking rule esteem in risk reagent, offered strategy by these analyst were not in base of situation and this model utilizing specifically of making greatest aggregate speculation productivity rate on the premise of quality in risk reagent. Likewise specialist offered another model for capital portion with utilizing of Monte Carlo simulation. Scientist execution of this offered model was this model can't grow up in spite of another premise on situation models [16].

Computation were done in risk reagent with utilizing of the parametric, for 100 dynamic organization in Tehran stock trade and they did expansion as an impediment to Markowitz sheets model and they composed distinctive improved portfolio with change in worth parameters in tolerating risk reagent in speculation and tolerating its certainty per penny. The manuscript result appeared, including esteem restriction in risk reagent to Markowitz model, maybe constrained helpful wilderness, changing to a spot as well as wantonness them.

Study was enhanced portfolio on the subject of dynamic organizations' sheets in nutritious businesses in Tehran stock trade with utilizing of worth as a part of risk reagent. Analyst utilized of sheets week by week statics of action organizations of nutritious ventures in stock trade done this examination. In the manuscript, they utilized of right arranging technique for making enhanced. Persistently there is decent result in this examination as specifically association between worth in risk reagent and industrialists desire productivity, in spite of the fact that there are not immediate connections between quality in risk reagent and assortment portfolio. 
Presentation was done model for picking advanced portfolio with utilizing of worth as a part of risk reagent and misuse of screenwriter model. In this exploration, first they did computation esteem in risk reagent's few sheets with utilizing playwright model and end of all; they selected putting streamlined manufactured model application in fear. Specialist achieved to presentation of picking portfolio model in stable advancing edge with utilizing esteem arrangement as a part of risk recommender.

\section{Top-Down Strength Period Presentation}

A standout amongst the most immediate and generally utilized utilizations of Modern portfolio theory is asset distribution. Since the benefit allotment choice is so imperative, all advantage supervisors and money related guides decide an ideal portfolio for their customers are they institutional or individual by playing out a benefit assignment examination utilizing an arrangement of benefit class. They start by selecting an arrangement of advantage classes for example local vast top and little top stocks, long haul bonds, global stocks. To get appraisals of the profits and volatilities and relationships, they for the most part begin with the verifiable execution of the files speaking to this benefit classes. These appraisals are utilized as inputs as a part of the mean-difference improvement which results in an effective outskirt. At that point, utilizing some measure for occasion, utilizing Monte Carlo reenactments to process the riches dispersions of the competitor portfolios, they pick an ideal portfolio. At long last, this portfolio is actualized utilizing either file or effectively overseen stores.

\section{Existing Study Differences On The MPT Refrain}

The essential asset portion model has been connected to numerous territories inside money. In this segment the author quickly depict four noteworthy applications that are gotten from Modern portfolio theory: asset distribution usage, component models and portfolio development, risk management by offer side firms, and overseeing dynamic risk. A use of Modern portfolio theory normally utilized by money related organizers is as a part of the usage of their customer's advantage assignment. The author found in the past area how Modern portfolio theory is connected in a top-down design to think of an ideal portfolio utilizing the normal execution of the advantage classes and the risk ravenousness of the customer. Nonetheless, rather than specifically putting assets into files that speak to the advantage class, execution is performed utilizing administrators with various styles that make up the benefit class.

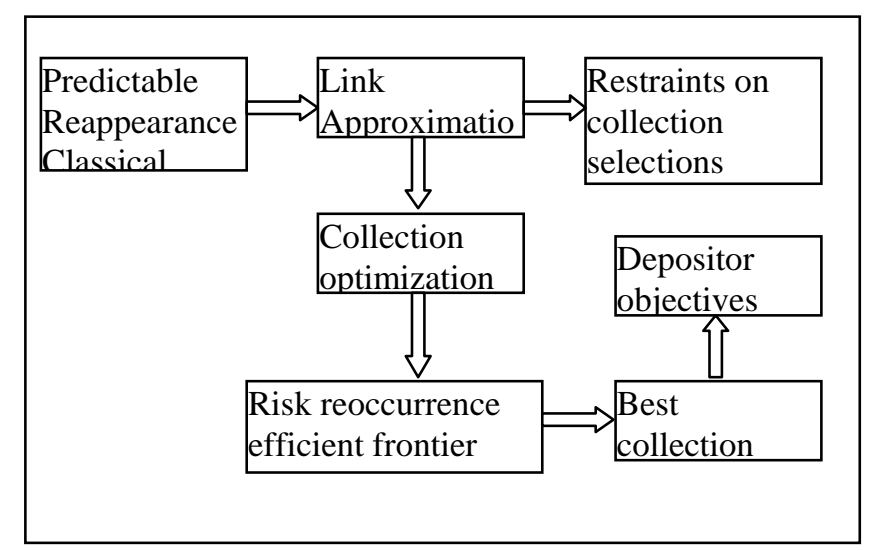

Fig. 1 The investment process of Modern portfolio theory.

Figure 1 shows a rundown of the Modern portfolio theory speculation process mean-fluctuation enhancement or the theory of portfolio determination. Despite the fact that the theory behind Modern portfolio theory is moderately straight forward, its usage can get entirely entangled. The theory 
manages that given assessments of the profits, volatilities, and connections of an arrangement of speculations and imperatives on venture decisions for instance, most extreme exposures and turnover requirements. It is conceivable to play out an improvement of outcomes in the risk/return or mean-difference proficient outskirts. This boondocks is effective on the grounds that basic each point on this wilderness is a portfolio that outcomes in the best conceivable expected return for that level of risk or results in the littlest conceivable risk for that level of expected return. The portfolios that lie on the outskirts make up the arrangement of productive portfolios.

\section{Selection of Collection: An Instance}

Utilizing an express case, the author now delineate how asset administrators and money related guides use Modern portfolio theory to assemble ideal portfolios for their customers. In this illustration the author will build a proficient boondocks made up of universal value, and shed some light on the determination of an ideal portfolio. The forward searching presumptions are designed for the four asset classes. These inputs are a case of evaluations that are not completely in light of authentic execution of these benefit classes. The normal return appraisals are made utilizing a risk premium approach that is getting the chronicled risk premiums appended to bonds, expansive top, mid-top, little top, and worldwide value and afterward have been subjectively modified to incorporate the advantage chief's assumptions with respect to the future long-run that could associate with five years to ten years execution of these benefit classes. The risk and connection figures are chiefly verifiable. The following stride is to utilize a product bundle to play out the improvement those outcomes in the effective boondocks.

\section{Handling Dynamic Risk or Chasing Fault Accounting}

For quite a while, institutional financial specialists have been working with asset directors in the quest for a system to deal with the dynamic risk connected with their portfolios in respect to a benchmark. The procedure, they trust, will allow them to spending plan, or apportion, risk over their dynamic directors. Additionally, if the approach is straightforward, so ex stake risk or return desires can be framed, then it is conceivable to reward financial specialists with the most extreme return for the level of risk embraced. At the end of the day, it is conceivable to proficiently assign the dynamic risk crosswise over supervisors, in this way settling on the dynamic designation choice productive. The way to building such a procedure lies in the verifiable relationship between the execution of dynamic administrators and the deviations from their portfolios benchmark. Generally as the way to building the arrangement of ideal portfolios lies in framing desires of the risk or return qualities of the different asset classes, understanding the primary or following blunder connections crosswise over various chiefs is the initial phase in making a following mistake planning strategy.

Table 1 Theoretical Dominant chasing fault performances of Managers of Large Capital

\begin{tabular}{|l|l|l|l|}
\hline $\begin{array}{l}\text { Predictable } \\
\text { Dominant }\end{array}$ & $\begin{array}{l}\text { Chasing } \\
\text { Fault }\end{array}$ & $\begin{array}{l}\text { Managers } \\
\text { of Large } \\
\text { capital }\end{array}$ & $\begin{array}{l}\text { Dominant } \\
\text { association }\end{array}$ \\
\hline $0 \%$ & $2 \%$ & Indexed & 1 \\
\hline $1 \%$ & $3 \%$ & Improved & .01 \\
\hline $2 \%$ & $5 \%$ & Essential & -.01 \\
\hline $3 \%$ & $6 \%$ & Dynamic & -.2 \\
\hline
\end{tabular}

The theoretical primary or following blunder connections for four sorts of residential expansive top value chiefs has been talked about here. The show additionally exhibits the relationships among the primary for the diverse directors. The listed administrator is not anticipated that would beat the benchmark and is additionally anticipated that would track the benchmark superbly. Subsequently, 
this kind of chief has a normal primary of zero with no following blunder. An improved filed or risk controlled administrator might be required to create a primary of $2 \%$ with a moderately low following mistake of $4 \%$. A risk controlled chief looks to create primary by making wagers just along one measurement, for example, the style, size, or division.

A center director could make wagers along more than one of those measurements, subsequently trying to create a bigger primary of $3 \%$ with a bigger following mistake of $5 \%$. At last, a more dynamic chief may play out some stock choice, thus would have the biggest dynamic risk a following mistake of $6 \%$ yet would likewise be required to produce the biggest primary. The connections are by and large supervisor particular, however in the event that chief styles are reciprocal for occasion if administrators are not looking for primary from the same sources, then the director primarys will in all likelihood be uncorrelated or even adversely associated. Not surprisingly, the most advantages from dynamic risk enhancement will originate from these sorts of supervisors. The proficient wilderness in following blunder space for this arrangement of supervisors has been examined. The effective portfolios change along the proficient outskirts, going from a $90 \%$ assignment to the listed administrator for no primary and following blunder to $90 \%$ apportioned to the dynamic chief for an primary of 5\% and a following mistake of $5 \%$. The ideal portfolios are talked about for a following blunder spending plan of 900 premise focuses. The purpose behind limiting the dynamic risk spending plan to such a little level is, to the point that for local vast capital value the arrival proportion falls after a following mistake of around 3\%. Subsequently, after that level the financial specialist gets a littler primary prize for every unit of following blunder.

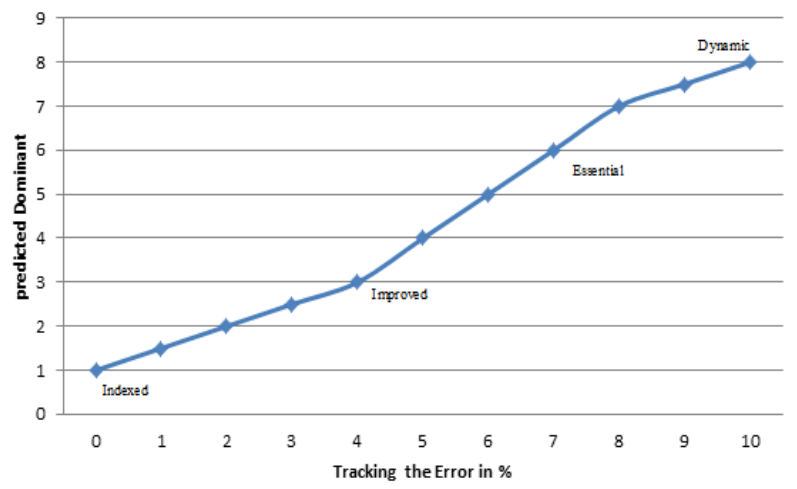

Fig. 1 Well-organized distribution of dynamic accomplishment that are with active risk.

\section{Conclusion}

At this point it is obvious that Modern portfolio theory, the theory initially explained, has discovered applications in numerous parts of modern money related theory and practice. The author has shown a couple of the most broadly utilized applications as a part of the zones of advantage assignment, portfolio management, and portfolio development. In spite of the fact that it took a couple of years to make a buzz, the late twenty-first hundreds of years saw no let-up in the spread of the utilization of Modern portfolio theory. Further, it is impossible that its prevalence will wind down at whatever time in the close or far off future. Therefore, it appears to be protected to foresee that Modern portfolio theory will possess a changeless spot in the theory and routine of money. Picking portfolio is one of the inquiry which to break out brain of most capital business sector dynamic individual. That which being particular diverse improvement portfolio model is standard of risk computation. Risk of contributing is one of the critical inquiry which entrepreneur facing on them. When in doubt, industrialist are trail obtaining to most productivity with least risk. Therefore one of the critical test to portfolio confining, is altering connection or streamlining weight of existing shares, in portfolio for making right for usage.

Ability models and extraordinarily expertise single model shaping on essential of new meaning of risk, this examination is utilizing of risk premonition and effectiveness in type of aptitude single 
model for portfolio enhancement. Congruity of definitions, scholarly entrepreneur being putting assets into one of these two figures: In venture position, financial specialist picking circumstance comparable risk with more proficiency. In venture position, financial specialist picking circumstance comparative proficiency with less risk.

Consequence of the manuscript is pleasant with second case. At the end of the day, despite the fact that the manuscript were demonstrate that decision portfolio effectiveness was comparative on the premise of greatest model of stable sharp with productivity of traditional model, yet have less risk and can great path in speculation action. Toward the end proposed under case to analyst: We can spend to examination foreknowledge effectiveness with real proficiency and premonition risk with genuine risk in advancement stable sharp model. Utilizing Genetic Algorithm to improve the sharp ratio and portfolio effectiveness, the anxious system behavior can be stabilized. Another model was investigated for the development of stable sharp ratios of portfolios, when the tradeoff of expected return and the risk is balanced.

\section{References}

[1] Abbasi, Ebrahim and et al, (2009), “Application of value in reagent (VAR) risk in formation of stock optimized portfolio in Tehran stock exchange”, Publication of researching economics, No 44.

[2] Abbasi, Ebrahim and et al, (2009), “Application of value introducer risk in framing optimized portfolio in Tehran stock exchange”, Publication of researching economics, No 87.

[3] Ahmadi, Mohamad vali; Sarmad, Majid, (2009), "Recognition of remote points in normal's amounts according to quantities (Improvement Observation)”, Journal of satisfaction. Volume 3, No 2.

[4] Babaie, Gholam reza and et al, (2007), "Methods of appointment outlying amounts in medical considerations", Journal of medical faculty, Tehran medical professional university, Period 65, September, No 7.

[5] Chen a Wei and et al,(2011), "Worst-case V a R and robust portfolio optimization with interval ran domun certainty set", Expert Systems with Applications", Vol. 38,Issue 1, January, pp 64-70.

[6] Fabbozi , Frank J and et al, (2007), "Robust Portfolio Optimization And Management ", Wiley.

[7] Farid, Dariush and et al, (2010), “Application of risk value and selection optimized portfolio using of Mont Karlou dramatist technique in Tehran stock exchange ”, Journal of knowledge expansion, eighteenth age, No 31.

[8] Fastrich , Björn and Winker ,Peter, (2009), "Robust Portfolio Optimization with a Hybrid Heuristic Algorithm", Computational Management Science.

[9] Gadiri Mogadam, Abolfazl; Rafie, Darani; (2010), “ Studying and making clear stock optimized portfolio in food active industrial companies in Tehran stock exchange ", Publication of economic and agriculture expansion, volume 24, No 3.

[10] Galloppo , Giuseppe,(2010),"A Comparison Of Pre And Post Modern Portfolio Theory Using Resampling ", Global Journal Of Business Research, Vol. 4, No. 1, pp. 1-16.

[11] Hanafizade, Payam and et al,(2006)," capital allocation with separation eventual space method”, searching scientific quarterly periodical of sharif, No 34.

[12] Khaluzade, Hamid; Amiri, Nasibeh,(2006), “ Appointment of optimized portfolio in IRAN stock exchange market according to value recommendation in risk reagent ",Journal of investigation economics, No 73.

[13] LuZhaosong, (2011), "Robust Portfolio Selection Based on a Joint Ellipsoidaluncertainty Set", Optimization Methods and Software, Vol. 26, Issue 1,p 89-104. 
[14] Natarajan, Karthik and et al, (2010), "Tractable Robust Expected Utility and Risk Modelsfor Portfolio Optimization", Mathematical Finance, Vol. 20, Issue 4,pp 695-731.

[15] Raeai, Reza; Saeedi, Ali; (2010),” Argument of financial and risk management engineering ”, Publications: SAMT, fifth edition, summer.

[16] Santos,André Alves Portela,(2010), "The Out-of-Sample Performance of Robust Portfolio Optimization", Brazilian Review of Finance, Vol. 8, No 2, pp141-166. 